\title{
Representations of Affine Multifunctions by Affine Selections
}

\author{
Valentin V. Gorokhovik
}

Received: 22 January 2008 / Accepted: 25 January 2008 /

Published online: 4 March 2008

(C) Springer Science + Business Media B.V. 2008

\begin{abstract}
The paper deals with affine selections of affine (both convex and concave) multifunctions acting between finite-dimensional real normed spaces. It is proved that each affine multifunction with compact values possesses an exhaustive family of affine selections and, consequently, can be represented by its affine selections. Moreover, a convex multifunction with compact values possesses an exhaustive family of affine selections if and only if it is affine. Thus the existence of an exhaustive family of affine selections is the characteristic feature of affine multifunctions which differs them from other convex multifunctions with compact values. Besides a necessary and sufficient condition for a concave multifunction to be affine on a given convex subset is also proved. Finally it is shown that each affine multifunction with compact values can be represented as the closed convex hull of its exposed affine selections and as the convex hull of its extreme affine selections. These statements extend the Straszewicz theorem and the Krein-Milman theorem to affine multifunctions.
\end{abstract}

Keywords Affine - Convex • Concave multifunction • Affine selections • Exposed and extremal selections $\cdot$ Krein-Milman theorem $\cdot$ Straszewicz theorem

Mathematics Subject Classifications (2000) $54 \mathrm{C} 60 \cdot 54 \mathrm{C} 65 \cdot$

Secondary $47 \mathrm{H} 04 \cdot 49 \mathrm{~J} 53$

Dedicated to Boris Mordukhovich in honour of his 60th birthday.

V. V. Gorokhovik $(\varangle)$

Institute of Mathematics, The National Academy of Sciences of Belarus,

Surganova st., 11, Minsk 220072, Belarus

e-mail: gorokh@im.bas-net.by 


\section{Introduction}

Let $X$ and $Y$ be two sets and let $F: X \rightrightarrows Y$ be a multivalued mapping or, shortly, a multifunction from $X$ into $Y$ which assigns to each $x \in X$ a (possibly empty) subset $F(x) \subset Y$. The sets

$$
\operatorname{dom} F:=\{x \in X \mid F(x) \neq \emptyset\}
$$

and

$$
\operatorname{gph} F:=\{(x, y) \in X \times Y \mid y \in F(x)\}
$$

are called the effective domain and the graph of the multifunction $F$ respectively.

A single-valued mapping $f: X \rightarrow Y$ is called a selection of a multifunction $F: X \rightrightarrows Y$ if $f(x) \in F(x)$ for all $x \in \operatorname{dom} F$.

A family $\mathscr{F}$ consisting of selections of a multifunction $F: X \rightrightarrows Y$ will be said to be exhaustive for $F$ if $F(x)=\{f(x) \mid f \in \mathscr{F}\}$ for all $x \in \operatorname{dom} F$.

The axiom of choice guarantees the existence of selections for any multifunctions with nonempty effective domain. However the existence of selections with desired properties like continuity, measurability, differentiability or some algebraic properties is not so trivial (see, for instance, [1-4, 6, 18-20, 27, 33, 36-42]).

In the paper we deal with affine selections of affine multifunctions acting from a finite-dimensional real normed space $X$ into another finite-dimensional real normed space $Y$ and taking compact values. It should be noted that different authors saying about affine multifunctions mean sometimes different things. When we say that a multifunction is affine we mean that it is both convex and concave. Affine multifunctions (under other title) were first studied by Gautier [7] and Lemarechal and Zowe [16] for the case when $X=\mathbb{R}$ and $\operatorname{dom} F=[0, T] \subset \mathbb{R}, T>0$. Affine multifunction acting between arbitrary finite-dimensional vector spaces was studied by Gorokhovik and Zabreiko [10,11]. These studies were motivated by attempts to extend to multifunctions classical notions of differentiability of functions based on local approximations with affine functions. Notice that another approach to differentiation of multifunctions when a derivative is defined as a multifunction whose graph is a tangent or normal (in one or another sense) cone to the graph of a multifunction that we differentiate is extensively developed now (see, for example, $[2,13,28-30,35])$. The realization of this approach based on the (limiting) normal cone was thoroughly developed by Mordukhovich [21-24], the most detail representation of these results can be found in his two-volume monograph [25, 26].

In this paper we continue the study of affine multifunctions originated in [8-11]. Here we focus our attention on representations of affine multifunctions by their affine selections. First we prove that each affine multifunction with compact values possesses an exhaustive family of affine selections and, consequently, can be represented by its affine selections. Moreover, we show that a convex multifunction with compact values possesses an exhaustive family of affine selections if and only if it is affine. Thus the existence of an exhaustive family of affine selections is the characteristic feature of affine multifunctions which differs them from other convex multifunctions with compact values.

As a subset of the normed vector space of single-valued affine functions from $X$ into $Y$ the family of all affine selections of an affine multifunction with compact values is convex and compact. It is natural to pose the converse question: when a 
given convex compact subset of single-valued affine functions is an exhaustive family of affine selections for some affine multifunction? To show that this question is not trivial we present an example of convex and compact subset of single-valued affine functions from $\mathbb{R}$ to $\mathbb{R}$ that is an exhaustive family of affine selections for the concave multifunction from $\mathbb{R}$ to $\mathbb{R}$ the restriction of which on any nontrivial interval of $\mathbb{R}$ is not affine. In the paper we obtain necessary and sufficient conditions for a convex and compact subset of single-valued affine functions to generate a multifunction that is affine on a given convex subset.

We introduce also the notion of exposed selections as well as the notion of extreme selections of a multifunction and prove that each affine multifunction with compact values can be represented as the closed convex hull of its exposed affine selections and as the convex hull of its extreme affine selections. These statements extend the Straszewicz theorem and the Krein-Milman theorem to affine multifunctions.

\section{Preliminaries}

Let $X$ and $Y$ be finite-dimensional normed vector spaces over reals $\mathbb{R}$.

A multifunction $\mathscr{A}: X \rightrightarrows Y$ is called

i.) convex if

$$
\alpha \mathscr{A}\left(x_{1}\right)+(1-\alpha) \mathscr{A}\left(x_{2}\right) \subset \mathscr{A}\left(\alpha x_{1}+(1-\alpha) x_{2}\right)
$$

for all $x_{1}, x_{2} \in \operatorname{dom} \mathscr{A}$ and $\alpha \in[0,1]$;

ii.) concave if dom $\mathscr{A}$ is a convex subset of $X$ and

$$
\alpha \mathscr{A}\left(x_{1}\right)+(1-\alpha) \mathscr{A}\left(x_{2}\right) \supset \mathscr{A}\left(\alpha x_{1}+(1-\alpha) x_{2}\right)
$$

for all $x_{1}, x_{2} \in \operatorname{dom} \mathscr{A}$ and $\alpha \in[0,1]$;

iii.) affine if

$$
\alpha \mathscr{A}\left(x_{1}\right)+(1-\alpha) \mathscr{A}\left(x_{2}\right)=\mathscr{A}\left(\alpha x_{1}+(1-\alpha) x_{2}\right)
$$

for all $x_{1}, x_{2} \in \operatorname{dom} \mathscr{A}$ and $\alpha \in[0,1]$.

It follows immediately from the above definition that a multifunction $\mathscr{A}: X \rightrightarrows Y$ is affine if and only if it is both convex and concave on dom $\mathscr{A}$.

Convex multifunctions were studied by many authors, see, for instance, $[2,17,31$, $32,34,35]$ and references therein. For concave multifunctions we can refer to the paper [12] devoted to fans (positively homogeneous and concave multifunctions). As it was noted above affine multifunctions were studied in [7-11,16].

Throughout the paper we will suppose that values of multifunctions involved in the study are convex and compact subsets of $Y$. As is known [14] the collection $\mathscr{K}(Y)$ of all compact convex subsets of $Y$ equipped with the addition (by Minkowski) and multiplication by nonnegative reals is a semilinear space [14]. The function

$$
d_{H}(M, N):=\inf \left\{\alpha \geqslant 0 \mid M \subset N+\alpha B_{Y}, N \subset M+\alpha B_{Y}\right\}
$$

( $B_{Y}$ is the unit ball in $Y$ ) called the Hausdorff distance determines a structure of a metric space on $\mathscr{K}(Y)$.

Thus a multifunction $F: X \rightrightarrows Y$ with compact convex values can be interpreted as the single-valued function from $\operatorname{dom} F \subset X$ into the semilinear metric space $\mathscr{K}(Y)$. 
Many concepts like continuity, uniform continuity, Lipschitz continuity and some others for such multifunctions coincide with analogous notions for single-valued mappings acting from one metric space to another.

In what follows we will denote multifunctions with compact and convex values by symbol $F: X \rightarrow \mathscr{K}(Y)$.

Given a multifunction $F: X \rightarrow \mathscr{K}(Y)$, the support function of $F$ is the real-valued function $s_{F}$ : $\operatorname{dom} F \times Y^{*} \rightarrow \mathbb{R}$ defined by

$$
s_{F}\left(x, y^{*}\right):=\max \left\{\left\langle y, y^{*}\right\rangle \mid y \in F(x)\right\} .
$$

Here $Y^{*}$ stands for a norm dual of $Y$.

It is easy to verify that a multifunction $\mathscr{A}: X \rightarrow \mathscr{K}(Y)$ is affine (respectively, convex or concave) if and only if for each $y^{*} \in Y^{*}$ the partial function $s_{\mathscr{A}}\left(\cdot, y^{*}\right)$ : $\operatorname{dom} \mathscr{A} \rightarrow \mathbb{R}$ is affine (respectively, concave or convex) on $Y^{*}$. For more detail dual characterizations of affine multifunctions we refer to the papers $[10,11]$.

Throughout this paper the collection of all affine multifunctions acting from $X$ into $Y$ and taking compact values will be denoted by $\mathfrak{A}(X, Y)$. The main aim of the paper is to study representations of affine multifunctions of $\mathfrak{A}(X, Y)$ by their affine selections.

By symbol $\mathbb{A}(X, Y)$ we will denote the vector space of single-valued affine functions from $X$ into $Y$. For $X$ and $Y$ are finite-dimensional the vector space $\mathbb{A}(X, Y)$ is also finite-dimensional. Since the vector space $\mathbb{A}(X, Y)$ is isomorphic to the cartesian product $\mathbb{L}(X, Y) \times Y$, where $\mathbb{L}(X, Y)$ stands for the vector space of linear mappings from $X$ into $Y$, we will present an element of $\mathbb{A}(X, Y)$ as a pair $(A, b)$ with $A \in \mathbb{L}(X, Y)$ and $b \in Y$. Besides we will suppose that some norm that is compatible with norms on $X$ and $Y$ is defined on $\mathbb{A}(X, Y)$. In particular, we can suppose that $\|(A, b)\|_{\mathbb{A}(X, Y)}:=\max \left\{\|A\|_{\mathbb{L}(X, Y)},\|b\|_{Y}\right\}$. Thus $\mathbb{A}(X, Y)$ is a finitedimensional normed space.

Given a multifunction $F: X \rightarrow \mathscr{K}(Y)$, the collection of all affine selections of $F$ will be denoted be $\mathscr{L}_{F}$. It is easy to verify that for any multifunction $F: X \rightarrow \mathscr{K}(Y)$ the collection $\mathscr{L}_{F}$ is a convex and closed (possibly, empty) subset of the normed vector space $\mathbb{A}(X, Y)$.

On the other hand, each nonempty compact convex subset $\mathscr{L}$ of $\mathbb{A}(X, Y)$ generates the concave multifunction $F_{\mathscr{L}}: X \rightrightarrows Y$ defined by

$$
F_{\mathscr{L}}(x)=\{A x+b \mid(A, b) \in \mathscr{L}\}
$$

with $\operatorname{dom} F_{\mathscr{L}}=X$ and $F_{\mathscr{L}}(x) \in \mathscr{K}(Y)$ for all $x \in X$.

\section{When is a Convex Multifunction Affine?}

The simplest sufficient condition for a convex multifunction with compact values to be affine is presented in the following proposition.

Proposition $1[10,11]$ Let $\mathscr{A}: X \rightrightarrows Y$ be a convex multifunction with compact values. If $\mathscr{A}\left(x^{0}\right)$ is a singleton (i.e. $\left.\mathscr{A}\left(x^{0}\right)=\left\{y^{0}\right\}\right)$ for some point $x^{0} \in \mathrm{ri}(\operatorname{dom} \mathscr{A})$ then $\mathscr{A}$ is actually a single-valued affine function on dom $\mathscr{A}$.

Here ri (dom $\mathscr{A})$ stands for the relative interior of dom $\mathscr{A}$.

悬 Springer 
The single-valuedness of convex multifunctions was also studied in [5].

The next assertion will used below to construct affine selections of affine multifunctions.

Proposition 2 For any affine multifunction $\mathscr{A} \in \mathfrak{A}(X, Y)$ and any $y^{*} \in Y^{*}$ the multifunction $\mathscr{A}\left(\cdot, y^{*}\right): X \rightrightarrows Y$, defined by

$$
\mathscr{A}\left(x, y^{*}\right):=\left\{y \in \mathscr{A}(x) \mid y^{*}(y)=\max _{z \in \mathscr{A}(x)} y^{*}(z)=s_{\mathscr{A}}\left(x, y^{*}\right)\right\},
$$

is also an affine multifunction of $\mathfrak{A}(X, Y)$ with $\operatorname{dom} \mathscr{A}\left(\cdot, y^{*}\right)=\operatorname{dom} \mathscr{A}$.

Proof Take any $y^{*} \in Y^{*}$. Since for every $x \in \operatorname{dom} \mathscr{A}$ the set $\mathscr{A}(x)$ is convex and compact and, consequently, $\mathscr{A}\left(x, y^{*}\right)$ is a nonempty, compact and convex subset of $Y$, we conclude that $\mathscr{A}\left(x, y^{*}\right) \in \mathscr{K}(Y)$ for all $x \in \operatorname{dom} \mathscr{A}\left(\cdot, y^{*}\right)$ and $\operatorname{dom} \mathscr{A}\left(\cdot, y^{*}\right)=$ $\operatorname{dom} \mathscr{A}$.

To prove that $\mathscr{A}\left(\cdot, y^{*}\right)$ is affine we need show that

$$
\alpha \mathscr{A}\left(x_{1}, y^{*}\right)+(1-\alpha) \mathscr{A}\left(x_{2}, y^{*}\right)=\mathscr{A}\left(\alpha x_{1}+(1-\alpha) x_{2}, y^{*}\right)
$$

for all $x_{1}, x_{2} \in \operatorname{dom} \mathscr{A}\left(\cdot, y^{*}\right)$ and $\alpha \in[0,1]$.

Since $\mathscr{A}\left(\alpha x_{1}+(1-\alpha) x_{2}, y^{*}\right) \subset \mathscr{A}\left(\alpha x_{1}+(1-\alpha) x_{2}\right)$ and $\alpha \mathscr{A}\left(x_{1}\right)+(1-\alpha) \mathscr{A}\left(x_{2}\right)=$ $\mathscr{A}\left(\alpha x_{1}+(1-\alpha) x_{2}\right)$ then for any $y \in \mathscr{A}\left(\alpha x_{1}+(1-\alpha) x_{2}, y^{*}\right)$ there exist $y_{1} \in \mathscr{A}\left(x_{1}\right)$ and $y_{2} \in \mathscr{A}\left(x_{2}\right)$ such that $y=\alpha y_{1}+(1-\alpha) y_{2}$. From $y_{1} \in \mathscr{A}\left(x_{1}\right)$ and $y_{2} \in \mathscr{A}\left(x_{2}\right)$ we obtain $y^{*}\left(y_{1}\right) \leqslant s_{\mathscr{A}}\left(x_{1}, y^{*}\right)$ and $y^{*}\left(y_{2}\right) \leqslant s_{\mathscr{A}}\left(x_{2}, y^{*}\right)$. Suppose that at least one of the latter two inequalities is strict. Then

$$
\begin{aligned}
y^{*}(y)= & \alpha y^{*}\left(y_{1}\right)+(1-\alpha) y^{*}\left(y_{2}\right)<\alpha s_{\mathscr{A}}\left(x_{1}, y^{*}\right)+ \\
& +(1-\alpha) s_{\mathscr{A}}\left(x_{2}, y^{*}\right)=s_{\mathscr{A}}\left(\alpha x_{1}+(1-\alpha) x_{2}, y^{*}\right),
\end{aligned}
$$

but it contradicts $y \in \mathscr{A}\left(\alpha x_{1}+(1-\alpha) x_{2}, y^{*}\right)$. It proves the inclusion

$$
\alpha \mathscr{A}\left(x_{1}, y^{*}\right)+(1-\alpha) \mathscr{A}\left(x_{2}, y^{*}\right) \supset \mathscr{A}\left(\alpha x_{1}+(1-\alpha) x_{2}, y^{*}\right) .
$$

The converse inclusion is proved in the similar way.

In the proof of the next theorem we will need the notion of an exposed point of a convex set.

Let $C$ be a convex set of a vector space $Y$. A point $y^{0} \in C$ is called an exposed point of $C$ if there exists a linear function $y^{*}: Y \rightarrow \mathbb{R}$ such that

$$
\left\{y \in C \mid y^{*}(y)=\max _{z \in C} y^{*}(z)\right\}=\left\{y^{0}\right\}
$$

or, in other words, if in the vector space $Y$ there exists a hyperplane that is support to $C$ at the point $y^{0} \in C$ and such that its intersection with $C$ consists of the only point $y^{0}$.

The collection of all exposed points of a convex set $C$ is denoted by $\exp C$. By the Straszewicz theorem $[15,34]$ for any compact convex set $C$ the subset of exposed points $\exp C$ is nonempty and $C=\operatorname{cl}(\operatorname{conv}(\exp C))$, where $\operatorname{cl} M$ and conv $M$ stand for the closure and the convex hull of a set $M$. 
Theorem 1 Any affine multifunction $\mathscr{A}: X \rightarrow \mathscr{K}(Y)$ admits affine selections.

Proof Let $x^{0} \in \mathrm{ri}(\operatorname{dom} \mathscr{A})$. Since $\mathscr{A}\left(x^{0}\right)$ is a nonempty compact convex subset of $Y$ then $\exp \mathscr{A}\left(x^{0}\right) \neq \emptyset$. Choose $\hat{y} \in \exp \mathscr{A}\left(x^{0}\right)$. By the definition of an exposed point there exists a linear function $\hat{y}^{*} \in Y^{*}$ such that $\{\hat{y}\}=\left\{y \in \mathscr{A}\left(x^{0}\right) \mid \hat{y}^{*}(y)=\right.$ $\left.s_{\mathscr{A}}\left(x^{0}, \hat{y}^{*}\right)\right\}$. From Proposition 2 we have that the multifunction

$$
\mathscr{A}\left(\cdot, \hat{y}^{*}\right): x \rightrightarrows\left\{y \in \mathscr{A}(x) \mid \hat{y}^{*}(y)=s_{\mathscr{A}}\left(x, \hat{y}^{*}\right)\right\}
$$

is affine and $\operatorname{dom} \mathscr{A}\left(\cdot, \hat{y}^{*}\right)=\operatorname{dom} \mathscr{A}$. Besides, since $x^{0} \in \operatorname{ri}\left(\operatorname{dom} \mathscr{A}\left(\cdot, \hat{y}^{*}\right)\right)$ and $\mathscr{A}\left(x^{0}, \hat{y}^{*}\right)=\{\hat{y}\}$, we conclude by Proposition 1 that a multifunction $\mathscr{A}\left(\cdot, \hat{y}^{*}\right): X \rightrightarrows Y$ is actually single-valued for all $x \in \operatorname{dom} \mathscr{A}$. Thus $\mathscr{A}\left(\cdot, \hat{y}^{*}\right)$ is a single-valued affine selection of $\mathscr{A}$.

Remark 1 If an affine function $\mathscr{A} \in \mathfrak{A}(X, Y)$ is also positively homogeneous then each affine selection of $\mathscr{A}$ is in fact a linear function. The existence of linear selections for positively homogeneous and affine multifunctions was proved by Zaslavski [42]. Thus Theorem 1 extends his result to arbitrary affine multifunctions of $\mathfrak{A}(X, Y)$.

Theorem 2 A convex multifunction $\mathscr{A}: X \rightrightarrows Y$ with compact values is affine if and only if in the space of single-valued affine mappings $\mathbb{A}(X, Y)$ there exists a nonempty compact convex subset $\mathscr{L}$ such that

$$
\mathscr{A}(x)=\{A x+b \mid(A, b) \in \mathscr{L}\} \quad \text { for all } \quad x \in \operatorname{dom} \mathscr{A}
$$

Proof Sufficiency. By the assumptions of the theorem a multifunction $\mathscr{A}: X \rightrightarrows Y$ is convex. On the other hand, the equality (2) implies that $\mathscr{A}$ coincides on dom $\mathscr{A}$ with the restriction of the concave multifunction $F_{\mathscr{L}}$ defined by (1). Thus, $\mathscr{A}$ is both convex and concave on dom $\mathscr{A}$ and, hence, it is affine.

Necessity. Without loss of generality we can suppose that $0 \in \mathrm{ri}(\operatorname{dom} \mathscr{A})$. Let $U$ be the linear hull of $\operatorname{dom} \mathscr{A}$ and let $P: X \rightarrow U$ be a projector of $X$ on $U$. Define the multifunction $\mathscr{A}_{1}: X \rightarrow \mathscr{K}(Y)$ by setting $\mathscr{A}_{1}(x)=\mathscr{A}(P x)$ for all $x \in X$. It follows immediately from the definition of $\mathscr{A}_{1}$ that dom $\mathscr{A}_{1}=\operatorname{dom} \mathscr{A}+\operatorname{ker} P$ and $0 \in \operatorname{int}\left(\operatorname{dom} \mathscr{A}_{1}\right)$. Here ker $P:=\{x \in X \mid P(x)=0\}$ stands for the kernel of $P$. It is not hard to see that $\mathscr{A}_{1}$ is an affine multifunction and $\mathscr{A}_{1}(x)=\mathscr{A}(x)$ for all $x \in \operatorname{dom} \mathscr{A}$ and, consequently, $\mathscr{L}_{\mathscr{A}_{1}} \subset \mathscr{L}_{\mathscr{A}}$.

Now we will show that $\mathscr{L}_{\mathscr{A}_{1}}$ is a bounded subset of the space of single-valued affine mappings $\mathbb{A}(X, Y)$.

Let $c(x):=\max _{y \in \mathscr{A}_{1}(x)}\|y\|$. Since for each $x \in \operatorname{dom} \mathscr{A}_{1}$ the value $\mathscr{A}_{1}(x)$ is a compact subset of $Y$, then $0<c(x)<+\infty$ for $x \in \operatorname{dom} \mathscr{A}_{1}$. By the definition of a selection we have $A x+b \in \mathscr{A}_{1}(x)$ for all $x \in \operatorname{dom} \mathscr{A}_{1}$ and, hence, $\|A x+b\| \leqslant c(x)$ for all $x \in \operatorname{dom} \mathscr{A}_{1}$ and for all $(A, b) \in \mathscr{L}_{\mathscr{A}_{1}}$. Consequently, for all $(A, b) \in \mathscr{L}_{\mathscr{A}_{1}}$ we have $\|b\| \leqslant c(0)$ and

$$
\|A x\| \leqslant\|A x+b\|+\|b\| \leqslant c(x)+c(0) \text { for all } x \in \operatorname{dom} \mathscr{A}_{1} .
$$


Since $0 \in \operatorname{int}\left(\operatorname{dom} \mathscr{A}_{1}\right)$ we conclude from the latter inequality by the BanachSteinhaus principle that there exists a real $C>0$ such that

$$
\|A\|_{\mathbb{L}(X, Y)} \leqslant C \quad \text { for all }(A, b) \in \mathscr{L}_{\mathscr{A}_{1}} .
$$

Thus

$$
\|(A, b)\|_{\mathbb{A}(X, Y)} \leqslant \max \{C, c(0)\} \quad \text { for all }(A, b) \in \mathscr{L}_{\mathscr{A}_{1}} .
$$

It proves that $\mathscr{L}_{\mathscr{A}_{1}}$ is a bounded subset of $\mathbb{A}(X, Y)$. As was noticed above $\mathscr{L}_{\mathscr{A}_{1}}$ is also closed and convex, therefore we conclude that $\mathscr{L}_{\mathscr{A}_{1}}$ is a compact convex subset of $\mathbb{A}(X, Y)$.

To complete the proof we need to show that $\mathscr{L}_{\mathscr{A}_{1}}$ is an exhaustive family of affine selections for $\mathscr{A}$. It follows from the proof of Theorem 1 that for each point $x \in$ int $\left(\operatorname{dom} \mathscr{A}_{1}\right)$ the set $\left\{A x+b \mid(A, b) \in \mathscr{L}_{\mathscr{A}_{1}}\right\}$ contains all exposed points of $\mathscr{A}_{1}(x)$. Consequently, by the Straszewicz theorem [15, Theorem 4.5] for each point $x \in \operatorname{int}\left(\operatorname{dom} \mathscr{A}_{1}\right)$ we have $\mathscr{A}_{1}(x)=\operatorname{cl}\left(\operatorname{conv}\left\{A x+b \mid(A, b) \in \mathscr{L}_{\mathscr{A}_{1}}\right\}\right)$. Since $\mathscr{L}_{\mathscr{A}_{1}}$ is a convex and compact subset of $\mathbb{A}(X, Y)$ the set $\left\{A x+b \mid(A, b) \in \mathscr{L}_{\mathscr{A}_{1}}\right\}$ is also convex and compact. Hence, $\mathscr{A}_{1}(x)=\left\{A x+b \mid(A, b) \in \mathscr{L}_{\mathscr{A}_{1}}\right\}$ for all $x \in \operatorname{int}\left(\operatorname{dom} \mathscr{A}_{1}\right)$. Due to the Hausdorff continuity of affine multifunctions [11, Proposition 3.13], the latter equality is extended to the whole effective domain dom $\mathscr{A}_{1}$. Thus $\mathscr{L}_{\mathscr{A}_{1}}$ is an exhaustive family of affine selections for $\mathscr{A}_{1}$ and, hence, for $\mathscr{A}$.

Corollary 1 For any affine multifunction $\mathscr{A} \in \mathfrak{A}(X, Y)$ there exists an exhaustive family of affine selections which is a compact convex subset of the normed vector space $\mathbb{A}(X, Y)$ of single-valued affine functions.

\section{When is a Concave Multifunction Affine?}

In this section we will study affine multifunctions as a subclass of concave multifunctions. Our aim is to find such characteristic features of affine multifunctions that differ them from other concave multifunctions.

From the results of the previous section we conclude that the necessary condition for any (including concave) multifunction to be affine is the existence of an exhaustive family of affine selections. Moreover, this family is a compact convex subset in the space of single-valued affine functions $\mathbb{A}(X, Y)$. However, as it shows the next example, this condition is not sufficient for a multifunction to be affine.

Example 1 The multifunction $F: \mathbb{R} \rightrightarrows \mathbb{R}$ with $\operatorname{gph} F:=\left\{(x, y) \mid y^{2}-x^{2} \leqslant 1\right\}$ is concave and the compact convex subset $\mathscr{L}_{F}=\left\{(a, b) \mid a^{2}+b^{2} \leqslant 1\right\}$ of $\mathbb{A}(\mathbb{R}, \mathbb{R})$ is the exhaustive family of affine selections of $F$. It is not difficult to see that the restriction of $F$ on any open interval of $\mathbb{R}$ is not affine.

Above observations motivate the following question.

Let $\mathscr{L}$ be a compact convex subset of $\mathbb{A}(X, Y)$ and let $F_{\mathscr{L}}$ be a multifunction defined by (1), $Q$ a nonempty convex subset of $X$. Under which conditions on $\mathscr{L}$ and $Q$ the restriction of the multifunction $F_{\mathscr{L}}$ on the set $Q$ is affine? 
For any $x \in X, y^{*} \in Y^{*}$ by symbol $\mathscr{L}\left(x, y^{*}\right)$ we denote a subset of $\mathscr{L}$ defined by

$$
\mathscr{L}\left(x, y^{*}\right):=\left\{(A, b) \in \mathscr{L} \mid y^{*}(A x+b)=\max _{\left(A^{\prime}, b^{\prime}\right) \in \mathscr{L}} y^{*}\left(A^{\prime} x+b^{\prime}\right)\right\} .
$$

Theorem 3 Let $\mathscr{L}$ be a compact convex subset of $\mathbb{A}(X, Y)$ and let $F_{\mathscr{L}}$ be the multifunction defined by (1). The restriction $F_{\mathscr{L}}$ on a nonempty convex subset $Q$ of $X$ is affine if and only if for any $y^{*} \in Y^{*}$ the inclusion $\mathscr{L}\left(x_{1}, y^{*}\right) \subset \mathscr{L}\left(x_{2}, y^{*}\right)$ holds for all $x_{1} \in \operatorname{ri} Q$, and $x_{2} \in Q$.

Proof Assume by contradiction that $F_{\mathscr{L}}$ is affine on $Q$ and $\mathscr{L}\left(x_{1}, y^{*}\right) \not \subset \mathscr{L}\left(x_{2}, y^{*}\right)$ for some $y^{*} \in Y^{*}$ and a couple of points $x_{1}, x_{2} \in Q$ with $x_{1} \in$ ri $Q$. Then for any $(A, b) \in \mathscr{L}\left(x_{1}, y^{*}\right)$ such that $(A, b) \notin \mathscr{L}\left(x_{2}, y^{*}\right)$ we have

$$
y^{*}\left(A x_{1}+b\right)=\max _{\left(A^{\prime}, b^{\prime}\right) \in \mathscr{L}^{\prime}} y^{*}\left(A^{\prime} x_{1}+b^{\prime}\right)=\max _{y \in F_{\mathscr{L}}\left(x_{1}\right)} y^{*}(y)
$$

and

$$
y^{*}\left(A x_{2}+b\right)<\max _{\left(A^{\prime}, b^{\prime}\right) \in \mathscr{L}} y^{*}\left(A^{\prime} x_{2}+b^{\prime}\right)=\max _{y \in F_{\mathscr{L}}\left(x_{2}\right)} y^{*}(y) .
$$

From $x_{1} \in \operatorname{ri} Q$ we have that $x_{1}=\alpha x_{2}+(1-\alpha) x_{2}^{\prime}$ for some $x_{2}^{\prime} \in Q$ and $\alpha \in(0,1)$. Since the multifunction $F_{\mathscr{L}}$ is affine we obtain the inequality

$$
\begin{aligned}
y^{*}\left(A x_{1}+b\right) & =\max _{y \in F_{\mathscr{L}}\left(x_{1}\right)} y^{*}(y)=\max _{y \in F_{\mathscr{L}}\left(\alpha x_{2}+(1-\alpha) x_{2}^{\prime}\right)} y^{*}(y) \\
& =\alpha \max _{y \in F_{\mathscr{L}}\left(x_{2}\right)} y^{*}(y)+(1-\alpha) \max _{y \in F_{\mathscr{L}}\left(x_{2}^{\prime}\right)} y^{*}(y) \\
& >\alpha y^{*}\left(A x_{2}+b\right)+(1-\alpha) y^{*}\left(A x_{2}^{\prime}+b\right) \\
& =y^{*}\left(A x_{1}+b\right)
\end{aligned}
$$

but it is impossible. The contradiction proves a necessary part of the theorem.

Now, let $\mathscr{L}$ be a compact convex subset of $\mathbb{A}(X, Y)$ such that for any $y^{*} \in Y^{*}$ the inclusion $\mathscr{L}\left(x_{1}, y^{*}\right) \subset \mathscr{L}\left(x_{2}, y^{*}\right)$ holds for all $x_{1} \in$ ri $Q$, and $x_{2} \in Q$.

As it was noted above for any compact convex subset $\mathscr{L}$ of $\mathbb{A}(X, Y)$ the multifunction $F_{\mathscr{L}}$ is concave and dom $F_{\mathscr{L}}=X$. Besides $F_{\mathscr{L}}$ is bounde d, Lipschitzian and uniformly continuous in the Hausdorff sense on the whole space $X$.

Let $x_{1}, x_{2}$ be arbitrary points of ri $Q$. Then $x_{\alpha}:=\alpha x_{1}+(1-\alpha) x_{2} \in \operatorname{ri} Q$ for all $\alpha \in[0,1]$. From the assumptions of the sufficient part of the theorem we obtain for all $y^{*} \in Y^{*}$ the equalities $\max _{y \in F \mathscr{L}\left(x_{\alpha}\right)} y^{*}(y)=\max _{y \in F_{\mathscr{L}}\left(x_{i}\right)} y^{*}(y), i=1$, 2. Consequently, for all $y^{*} \in Y^{*}$ we have $s_{F_{\mathscr{L}}}\left(x_{\alpha}, y^{*}\right)=\max _{y \in F_{\mathscr{L}}\left(x_{\alpha}\right)} y^{*}(y)=\alpha \max _{y \in F_{\mathscr{L}}\left(x_{1}\right)} y^{*}(y)+$ $(1-\alpha) \max _{y \in F_{\mathscr{L}}\left(x_{2}\right)} y^{*}(y)=\alpha s_{F_{\mathscr{L}}}\left(x_{1}, y^{*}\right)+(1-\alpha) s_{F_{\mathscr{L}}}\left(x_{2}, y^{*}\right)$. Thus $F_{\mathscr{L}}\left(x_{\alpha}\right)=$ $\alpha F_{\mathscr{L}}\left(x_{1}\right)+(1-\alpha) F_{\mathscr{L}}\left(x_{2}\right)$ for all $x_{1}, x_{2} \in$ ri $Q$ and $\alpha \in[0,1]$. It follows immediately from the definition that the multifunction $F_{\mathscr{L}}$ is affine on ri $Q$. Since $F_{\mathscr{L}}$ is Hausdorff continuous it is affine on the whole set $Q$. 
Corollary 2 A concave multifunction $\mathscr{A} \in \mathfrak{A}(X, Y)$ is affine if and only if in the space of single-valued affine mappings $\mathbb{A}(X, Y)$ there exists a nonempty compact convex subset $\mathscr{L}$ such that

$$
\mathscr{A}(x)=\{A x+b \mid(A, b) \in \mathscr{L}\} \quad \text { for all } \quad x \in \operatorname{dom} \mathscr{A}
$$

and, moreover, for any $y^{*} \in Y^{*}$ the inclusion $\mathscr{L}\left(x_{1}, y^{*}\right) \subset \mathscr{L}\left(x_{2}, y^{*}\right)$ holds for all $x_{1} \in \operatorname{ri}(\operatorname{dom} \mathscr{A}), x_{2} \in \operatorname{dom} \mathscr{A}$.

\section{Exposed and Extreme Affine Selections of Affine Multifunctions}

Let $C$ be a convex set. A point $y \in C$ is called an extreme point of $C$ provided for any two points $c, d \in C$ such that $(c+d) / 2=y$ one has $y=c=d$. The subset of all extreme points of $C$ is denoted ext $C$.

As is well-known [34] each exposed point of $C$ is an extreme point. Thus, $\exp C \subset \operatorname{ext} C$. Notice, that the latter inclusion is proper, in general. Besides we recall that by the Krein-Milman theorem for a compact convex set $C$ of a finite-dimensional space one has $C=\operatorname{conv}(\operatorname{ext} C)$.

We say that a selection $f: X \rightarrow Y$ of a multifunction $F: X \rightarrow \mathscr{K}(Y)$ is exposed (respectively, extreme) if $f(x) \in \exp F(x)$ [respectively, $f(x) \in \operatorname{ext} F(x)$ ] for all $x \in \operatorname{dom} F$.

In this section we show that, like compact convex sets, affine multifunctions of $\mathfrak{A}(X, Y)$ are entirely characterized by their exposed and extreme affine selections.

Theorem 4 For any affine multifunction $\mathscr{A} \in \mathfrak{A}(X, Y)$ the set of all its exposed affine selections Exp $\mathscr{A}$ is nonempty and

$$
\mathscr{A}(x)=\operatorname{cl}(\operatorname{conv}\{A x+b \mid(A, b) \in \operatorname{Exp} \mathscr{A}\}) \quad \text { for all } \quad x \in \operatorname{dom} \mathscr{A} .
$$

Proof Let $\mathscr{A} \in \mathfrak{A}(X, Y)$ be an affine multifunction and let $\hat{x} \in \operatorname{ri}(\operatorname{dom} \mathscr{A})$ and $\hat{y} \in \exp \mathscr{A}(\hat{x})$. It was shown in the proof of Theorem 2 that there exist $\hat{y}^{*} \in Y^{*}, \hat{y}^{*} \neq 0$, and an affine selection $(A, b)$ of the multifunction $\mathscr{A}$ such that $\hat{y}=A \hat{x}+b$ and $\mathscr{A}\left(x, \hat{y}^{*}\right)=\{A x+b\}$ for all $x \in \operatorname{dom} \mathscr{A}$. From the latter equality and definitions of an exposed point and of the multifunction $\mathscr{A}\left(x, \hat{y}^{*}\right)$ we conclude that $(A, b)$ is an exposed affine selection of the affine multifunction $\mathscr{A}$.

Thus we see that $\operatorname{Exp} \mathscr{A} \neq \emptyset$. Moreover, for any point $x \in \operatorname{ri}(\operatorname{dom} \mathscr{A})$ and $y \in \exp \mathscr{A}(x)$ there exists an exposed affine selection $(A, b) \in \operatorname{Exp} \mathscr{A}$ such that $y=A x+b$. By Straszewicz's theorem we obtain that $\mathscr{A}(x)=\operatorname{cl}(\operatorname{conv}\{A x+$ $b \mid(A, b) \in \operatorname{Exp} \mathscr{A}\})$ for all $x \in \operatorname{ri}(\operatorname{dom} \mathscr{A})$. Since $\mathscr{A}$ is Hausdorff continuous this equality is extended to all $x \in \operatorname{dom} \mathscr{A}$.

The next corollary was actually proved in the proof of the above theorem.

Corollary 3 An affine selection $(A, b): x \rightarrow A x+b$ of an affine multifunction $\mathscr{A} \in$ $\mathfrak{A}(X, Y)$ is exposed if and only if $A x^{0}+b \in \exp \mathscr{A}\left(x^{0}\right)$ at least for one point $x^{0} \in \operatorname{ri}(\operatorname{dom}(\mathscr{A})$. 
Now we prove the criterium for recognizing the extreme points of convex sets. We shall need this criterium in proving the theorem on a representation of an affine multifunction with its extreme affine selections.

Proposition 3 Let $C$ be a convex subset of the finite-dimensional space $Y$ with $\operatorname{dim} C \geqslant 1$. A point $y^{0} \in C$ is an extreme point of $C$ if and only if there exists an finite ordered family of linearly independent linear functions $\left\{y_{1}^{*}, y_{2}^{*}, \ldots, y_{k}^{*}\right\} \subset Y^{*}$, $1 \leqslant k \leqslant \operatorname{dim} C$, such that $y^{0}$ is a unique point of $C$ satisfying the following equalities

$$
y_{i}^{*}\left(y^{0}\right)=\max _{z \in C_{i-1}} y_{i}^{*}(z), i=1,2, \ldots, k,
$$

where $C_{0}=C, C_{i}=\left\{y \in C_{i-1} \mid y_{i}^{*}(y)=\max _{z \in C_{i-1}} y_{i}^{*}(z)\right\}, i=1,2, \ldots, k-1$.

Proof Sufficiency. Let a point $y^{0} \in C$ be a unique point of $C$ satisfying the equalities (3) where $\left\{y_{1}^{*}, y_{2}^{*}, \ldots, y_{k}^{*}\right\} \subset Y^{*}$ is an finite ordered family of linearly independent linear functions of $Y^{*}$. It immediately follows from the definition of an exposed point that $y^{0}$ is an exposed and, hence, extreme point of the set $C_{k-1}$. Since $C_{k-1}$ is the intersection of $C_{k-2}$ with the hyperplane $H_{k-2}:=\left\{y \in Y \mid y_{k-2}^{*}(y)=\max _{z \in C_{k-2}} y_{k-2}^{*}(z)\right\}$ supporting to $C_{k-2}$ we can conclude by Lemma 4.1 of [15] that $y^{0} \in \operatorname{ext} C_{k-2}$. In turn, $C_{k-2}$ is the intersection of $C_{k-3}$ with the hyperplane $H_{k-2}:=\left\{y \in Y \mid y_{k-3}^{*}(y)=\right.$ $\left.\max _{z \in C_{k-3}} y_{k-3}^{*}(z)\right\}$ supporting to $C_{k-3}$ and, hence, $y^{0} \in \operatorname{ext} C_{k-3}$. Arguing further in such a way we shall get on the $(k-1)$-th stage that $y^{0} \in \operatorname{ext} C$.

Necessity. It is easily seen that an extreme point $y^{0}$ of a convex set $C$ also is a relatively boundary point of $C$. Consequently (see, for instance, [34, Corollary 11.6.2]), there exists a linear function $y_{1}^{*} \in Y^{*}, y_{1}^{*} \neq 0$, that is not constant on $C$ and achieves a maximum over $C$ at the point $y^{0}$. Thus, for $C_{1}:=\left\{y \in C \mid y^{*}(y)=\right.$ $\left.\max _{z \in C} y_{1}^{*}(z)\right\}$ we have $\operatorname{dim} C_{1}<\operatorname{dim} C$ and $y^{0} \in C_{1}$.

When $C_{1}=\left\{y^{0}\right\}$, the one-element family $\left\{y_{1}^{*}\right\}$ is a desired ordered family of linear functions and the proof is complete.

In the case when $C_{1} \neq\left\{y^{0}\right\}$ we shall have that $y^{0} \in \operatorname{ext} C_{1}$ (see, for instance, Lemma 4.1 of [15]) and we can continue to construct a desired family of linear functions. To this end we choose a linear function $y_{2}^{*} \in Y^{*}$ that is not constant on $C_{1}$ and achieves a maximum over $C_{1}$ at the point $y^{0}$. Notice that, since $y_{2}^{*}$ is not constant on $C_{1}$ whereas $y_{1}^{*}$ is constant on $C_{1}$, the linear functions $y_{1}^{*}, y_{2}^{*}$ are linearly independent. Besides,

$$
y_{i}^{*}\left(y^{0}\right)=\max _{z \in C_{i-1}} y_{i}^{*}(z), i=1,2, \quad\left(C_{0}=C\right)
$$

and for $C_{2}:=\left\{y \in C_{1} \mid y^{*}(y)=\max _{z \in C_{1}} y_{1}^{*}(z)\right\}$ we have $\operatorname{dim} C_{2}<\operatorname{dim} C_{1}$ and $y^{0} \in C_{2}$.

Provided $C_{2}=\left\{y^{0}\right\}$, the ordered two-element family $\left\{y_{1}^{*}, y_{2}^{*}\right\}$ is a desired family of linear functions and the proof is complete.

In the case when $C_{2} \neq\left\{y^{0}\right\}$ we shall continue the process of the construction of the desired family. Since $1 \leqslant \operatorname{dim} C \leqslant \operatorname{dim} Y<+\infty$ we shall get on some $k$-th stage with $1 \leqslant k \leqslant \operatorname{dim} C$ the ordered linearly independent family $y_{1}^{*}, y_{2}^{*}, \ldots, y_{k}^{*}$ of linear functions satisfying (3) and such that $C_{k}=\left\{y^{0}\right\}$. Thus the desired family will be constructed. 
Theorem 5 For any affine multifunction $\mathscr{A} \in \mathfrak{A}(X, Y)$ the set of all its extreme affine selections Ext $\mathscr{A}$ is nonempty and

$$
\mathscr{A}(x)=\operatorname{conv}\{A x+b \mid(A, b) \in \text { Ext } \mathscr{A}\} \quad \text { for all } x \in \operatorname{dom} \mathscr{A} .
$$

Proof Since $\operatorname{Exp} \mathscr{A} \subset$ Ext $\mathscr{A}$ and by Theorem 4 Exp $\mathscr{A} \neq \emptyset$, we also have Ext $\mathscr{A} \neq \emptyset$.

To prove the equality (4) we associate with each finite ordered family of linear functions $\left\{y_{1}^{*}, y_{2}^{*}, \ldots, y_{k}^{*}\right\} \subset Y^{*}$ the family of the multifunctions

$$
\left\{\mathscr{A}\left(\cdot, y_{1}^{*}\right), \mathscr{A}\left(\cdot, y_{1}^{*}, y_{2}^{*}\right), \ldots, \mathscr{A}\left(\cdot, y_{1}^{*}, y_{2}^{*}, \ldots, y_{k}^{*}\right)\right\}
$$

defined by

$$
\begin{gathered}
\mathscr{A}\left(x, y_{1}^{*}\right):=\left\{y \in \mathscr{A}(x) \mid y_{1}^{*}(y)=\max _{z \in \mathscr{A}(x)} y_{1}^{*}(z)\right\} \\
\mathscr{A}\left(x, y_{1}^{*}, y_{2}^{*}\right):=\left\{y \in \mathscr{A}\left(x, y_{1}^{*}\right) \mid y_{2}^{*}(y)=\max _{z \in \mathscr{A}\left(x, y_{1}^{*}\right)} y_{2}^{*}(z)\right\}
\end{gathered}
$$

$\mathscr{A}\left(x, y_{1}^{*}, y_{2}^{*}, \ldots, y_{k}^{*}\right):=\left\{y \in \mathscr{A}\left(x, y_{1}^{*}, y_{2}^{*}, \ldots, y_{k-1}^{*}\right) \mid y_{k}^{*}(y)=\max _{z \in \mathscr{A}\left(x, y_{1}^{*}, y_{2}^{*}, \ldots, y_{k-1}^{*}\right)} y_{k}^{*}(z)\right\}$.

It follows from Proposition 2 that all multifunctions of this family are affine and the effective domain of each of them coincides with dom $\mathscr{A}$. Besides it is immediate from the above definition that

$$
\mathscr{A}\left(x, y_{1}^{*}, y_{2}^{*}, \ldots, y_{k}^{*}\right) \subset \mathscr{A}\left(x, y_{1}^{*}, y_{2}^{*}, \ldots, y_{k-1}^{*}\right) \subset \ldots \mathscr{A}\left(x, y_{1}^{*}\right) \subset \mathscr{A}(x)
$$

for $x \in \operatorname{dom} \mathscr{A}$.

Let $\mathscr{L}_{\mathscr{A}}$ as was denoted above be the collection of all affine selections of a multifunction $\mathscr{A}$ and let $x^{0} \in$ ri $(\operatorname{dom} \mathscr{A})$ and $y^{0} \in \operatorname{ext} \mathscr{A}\left(x^{0}\right)$. By Theorem 1 there exists an affine selection $(A, b) \in \mathscr{L}_{\mathscr{A}}$ such that $y^{0}=A x^{0}+b$. Since $y^{0} \in \operatorname{ext} \mathscr{A}\left(x^{0}\right)$ we can claim by the criterium proved in Proposition 3 that there exists an ordered linearly independent family of linear functions $\left\{y_{1}^{*}, y_{2}^{*}, \ldots, y_{k}^{*}\right\} \subset Y^{*}, 1 \leqslant k \leqslant \operatorname{dim} \mathscr{A}\left(x^{0}\right)$, satisfying the equality $\mathscr{A}\left(x^{0}, y_{1}^{*}, y_{2}^{*}, \ldots, y_{k}^{*}\right)=\left\{A x^{0}+b\right\}$. It is immediate from the inclusions (5) that $\left\{A x^{0}+b\right\} \in \mathscr{A}\left(x^{0}, y_{1}^{*}, y_{2}^{*}, \ldots, y_{i}^{*}\right), i=1,2, \ldots, k$.

Thus we have that $\left\{A x^{0}+b\right\} \in \mathscr{A}\left(x^{0}, y_{1}^{*}\right)$. Comparing the definitions of $\mathscr{A}\left(x^{0}, y_{1}^{*}\right)$ and of $\mathscr{L}_{\mathscr{A}}\left(x^{0}, y^{*}\right)$ we can see that $A x^{0}+b \in \mathscr{A}\left(x^{0}, y_{1}^{*}\right)$ is equivalent to $(A, b) \in \mathscr{L}_{\mathscr{A}}\left(x^{0}, y^{*}\right)$. Since $x^{0} \in \mathrm{ri}(\operatorname{dom} \mathscr{A})$, we conclude from Theorem 3 that $\mathscr{L}_{\mathscr{A}}\left(x^{0}, y^{*}\right) \subset \mathscr{L}_{\mathscr{A}}\left(x, y^{*}\right)$ for all $x \in \operatorname{dom} \mathscr{A}$ and, hence, $(A, b) \in \mathscr{L}_{\mathscr{A}}\left(x, y^{*}\right)$ for all $x \in \operatorname{dom} \mathscr{A}$ or, equivalently, $A x+b \in \mathscr{A}\left(x, y_{1}^{*}\right)$ for all $x \in \operatorname{dom} \mathscr{A}$. Thus $(A, b)$ is an affine selection of the multifunction $\mathscr{A}\left(\cdot, y_{1}^{*}\right)$.

Arguing in the analogous manner we deduce from $\left\{A x^{0}+b\right\} \in \mathscr{A}\left(x^{0}, y_{1}^{*}, y_{2}^{*}\right)$ and $x^{0} \in \operatorname{ri}(\operatorname{dom} \mathscr{A})$ that $A x+b \in \mathscr{A}\left(x, y_{1}^{*}, y_{2}^{*}\right)$ for all for all $x \in \operatorname{dom} \mathscr{A}$ or, equivalently, that $(A, b)$ is an affine selection of the multifunction $\mathscr{A}\left(\cdot, y_{1}^{*}, y_{2}^{*}\right)$.

Appealing to analogous arguments we shall obtain on the $k$-th stage that $A x+b \in$ $\mathscr{A}\left(x, y_{1}^{*}, y_{2}^{*}, \ldots, y_{k}^{*}\right)$ for all for all $x \in \operatorname{dom} \mathscr{A}$. Since $\mathscr{A}\left(x^{0}, y_{1}^{*}, y_{2}^{*}, \ldots, y_{k}^{*}\right)=$ 
$\left\{A x^{0}+b\right\}$ and $x^{0} \in \operatorname{ri}(\operatorname{dom} \mathscr{A})$, it follows from Proposition 1 that the multifunction $\mathscr{A}\left(\cdot, y_{1}^{*}, y_{2}^{*}, \ldots, y_{k}^{*}\right)$ is, in fact, single-valued and, hence, $\mathscr{A}\left(x, y_{1}^{*}, y_{2}^{*}, \ldots, y_{k}^{*}\right)=$ $\{A x+b\}$ for all $x \in \operatorname{dom} \mathscr{A}$.

Thus, for each $x \in \operatorname{dom} \mathscr{A}$ the point $y=A x+b$ is the unique point of $\mathscr{A}(x)$ which satisfies the equalities (5) with $C=\mathscr{A}(x)$. Consequently, it follows from the criterium proved in Proposition 3 that $A x+b \in \operatorname{ext} \mathscr{A}(x)$ for all $x \in \operatorname{dom} \mathscr{A}$, that is $(A, b)$ : $X \rightarrow Y$ is an extreme affine selection of the multifunction $\mathscr{A}$.

Thus, for any point $x \in \operatorname{ri}(\operatorname{dom} \mathscr{A})$ and $y \in \operatorname{ext} \mathscr{A}(x)$ there exists an extreme affine selection $(A, b) \in E x t \mathscr{A}$ such that $y=A x+b$. By the Krein-Milman theorem we obtain that $\mathscr{A}(x)=\operatorname{conv}\{A x+b \mid(A, b) \in E x t \mathscr{A}\}$ for all $x \in \operatorname{ri}(\operatorname{dom} \mathscr{A})$. Since $\mathscr{A}$ is Hausdorff continuous this equality is extended to all $x \in \operatorname{dom} \mathscr{A}$.

The following corollary immediately follows from the proof of the above theorem.

Corollary 4 An affine selection $(A, b): x \rightarrow A x+b$ of an affine multifunction $\mathscr{A} \in \mathfrak{A}(X, Y)$ is extreme if and only if $A x^{0}+b \in \operatorname{ext} \mathscr{A}\left(x^{0}\right)$ at least for one point $x^{0} \in \operatorname{ri}(\operatorname{dom}(\mathscr{A})$.

Example $2[7,11,16]$. Let $X=\mathbb{R}, Y=\mathbb{R}^{2}$. The multifunction $\mathscr{A}: \mathbb{R} \rightrightarrows \mathbb{R}^{2}$ of $\mathfrak{A}\left(\mathbb{R}, \mathbb{R}^{2}\right)$ defined by

$$
\operatorname{gph} \mathscr{A}=\left\{(x, y) \in \mathbb{R} \times \mathbb{R}^{2}|| y_{1}|\leqslant 1-x,| y_{2} \mid \leqslant 1+x\right\}
$$

is affine on its effective domain dom $\mathscr{A}=[-1,1]$. There are exactly four extreme affine selections of the multifunction $\mathscr{A}$ :

$$
\begin{gathered}
\left(A_{1}, b_{1}\right) ; x \rightarrow(-x+1,-x-1) ;\left(A_{2}, b_{2}\right) ; x \rightarrow(-x+1, x+1) \\
\left(A_{3}, b_{3}\right) ; x \rightarrow(x-1,-x-1) ;\left(A_{4}, b_{4}\right) ; x \rightarrow(x-1, x+1) .
\end{gathered}
$$

Consequently, by Theorem 4 we have

$$
\mathscr{A}(x)=\operatorname{conv}\{(-x+1,-x-1),(-x+1, x+1),(x-1,-x-1),(x-1, x+1)\}
$$

for all $x \in[-1,1]$.

Notice that the multifunction

$$
\mathscr{P}: x \rightrightarrows \operatorname{conv}\{(-x+1,-x-1),(-x+1, x+1),(x-1,-x-1),(x-1, x+1)\}
$$

is concave on the whole line $\mathbb{R}$ whereas its restriction to each of three intervals $[-\infty,-1],[-1,1]$ and $[1,+\infty]$ is affine.

Acknowledgements The author thanks an anonymous referee for his valuable remarks and suggestions.

The research was supported by the National Program of Fundamental Researches of Belarus under grant "Mathematical Models-16".

\section{References}

1. Artstein, Z.: On the calculus of set-valued maps and set-valued evolution equations. Set-Valued Anal. 3, 213-261 (1995) 
2. Aubin, J.-P., Frankowska, H.: Set-Valued Analysis. Birkhauser, Boston (1990)

3. Castaing, C., Valadier, M.: Convex Analysis and Measurable Multifunctions. Lecture Notes in Mathematics, vol. 580. Springer-Verlag, Berlin (1977)

4. Dentcheva, D.: Defferentiable selections and castaing representations of multifunctions. J. Math. Anal. Appl. 223, 371-396 (1998)

5. Deutsch, F., Singer, I.: On single-valuedness of convex set-valued maps. Set-Valued Anal. 1(1), 97-103 (1998)

6. Fintzel, M., Li, W.: Piecewise affine selections for piecewise polyhedral multifunctions and metric projections. J. Convex Anal. 7(1), 73-94 (2000)

7. Gautier, S.: Affine and eclipsing multifunctions. Numer. Funct. Anal. Appl. 11, 679-699 (1990)

8. Gorokhovik, V.V., Kishchenko, M.G.: On affine selections of multivalued mappings. Trudy Inst. Mat. NAN Belarusi 5, 51-54 (2000) (in Russian)

9. Gorokhovik, V.V., Kishchenko, M.G.: Affine selections of affine multivalued mappings. Vesti Natsijanal'naj akademii nawuk Belarusi. Ser. fizika-matematychnukh nawuk, (No. 1) 51-54 (2002) (in Russian)

10. Gorokhovik, V.V., Zabreiko, P.P.: Differentiability of multivalued mappings in sense of Fréchet. Trudy Inst. Mat. NAN Belarusi 1, 34-49 (1998) (in Russian)

11. Gorokhovik, V.V., Zabreiko, P.P.: On Fréchet differentiability of multifunctins. Optimization 54, 391-409 (2005)

12. Ioffe, A.D.: Nonsmooth analysis: differential calculus of nondifferentiable mappings. Trans. Amer. Math. Soc. 266, 1-56 (1981)

13. Kruger, A.Y.: Strict $\varepsilon$-subdifferentials and differentiation of set-valued mappings. Dokl. Akad. Nauk Belarusi 40(6), 38-43 (1996) (in Russian)

14. Kutateladze, S.S., Rubinov, A.M.: Minkowski Duality and its Applications. Nauka, Novosibirsk (1976) (in Russian)

15. Leichtweiss, K.: Konvexe Mengen. VEB Deutscher Verlag der Wissenschaften, Berlin (1980)

16. Lemarechal, C., Zowe, J.: The eclipsing concept to approximate a multi-valued mapping. Optimization 22, 3-37 (1991)

17. Makarov, V.L., Rubinov, A.M.: Mathematical Theory of Economic Dynamics and Equilibrium. Springer-Verlag, New York (1979)

18. Michael, E.A.: Continuous selection I. Ann. Math. 63(2), 361-382 (1956)

19. Michael, E.A.: Continuous selection II. Ann. Math. 64(3), 562-580 (1956)

20. Michael, E.A.: Continuous selection III. Ann. Math. 65(2), 375-390 (1957)

21. Mordukhovich, B.S.: Approximation Methods in Problems of Optimization and Control. Nauka, Moscow (1988) (in Russian)

22. Mordukhovich, B.S.: Generalized differential calculus for nonsmooth and set-valued mappings. J. Math. Anal. Appl. 183, 250-288 (1994)

23. Mordukhovich, B.S.: Nonconvex differential calculus for infinite-dimensional multifunctions. Set-Valued Anal. 4, 205-236 (1996)

24. Mordukhovich, B.S.: Coderivatives of set-valued mappings: calculus and applications. Nonlinear Anal. 30, 3059-3070 (1997)

25. Mordukhovich, B.S.: Variational Analysis and Generalized Differentiation, I: Basic Theory. Grundlehren Series (Fundamental Principles of Mathematical Sciences), vol. 330. Springer, Berlin (2006)

26. Mordukhovich, B.S.: Variational Analysis and Generalized Differentiation, I: Applications. Grundlehren Series (Fundamental Principles of Mathematical Sciences), vol. 331. Springer, Berlin (2006)

27. Nikodem, K.: Additive selections of additive set-valued functions. Univ. u Novom Sadu, Zb. Rad. Prirod.-Mat. Fak., Ser. Mat. 18(1), 143-148 (1988)

28. Penot, J.-P.: Differentiability of relations and differential stability of perturbed optimization problems. SIAM J. Control Optim. 22, 529-551 (1984)

29. Polovinkin, E.S.: The Theory of Multivalued Mappings. Moscow Physicotechnical Institute, Moscow (1986)

30. Polovinkin, E.S., Smirnov, G.V.: Differentiation of multivalued mappings and properties of solutions of differential inclusions. Soviet Math. Doklady 33, 662-666 (1986)

31. Pshenichnyi, B.N.: Convex multivalued mappings and their conjugates. In: Łoś, J., Łoś, M.W. (eds.) Mathematical Models in Economics, pp. 333-349. PWN-Polish Scientific Publishers, Warszawa (1974) 
32. Pshenichnyi, B.N.: Convex Analysis and Extremum Problems. Nauka, Moscow (1980) (in Russian)

33. Przeslawski, Krz.: Linear and lipschitz continuous selectors for the family of convex sets in euclidian vector spaces. Bull. Polish Acad. Sci. Math. 33(1-2), 31-34 (1985)

34. Rockafellar, R.T.: Convex Analysis. Princeton University Press, Princeton, New Jersey (1970)

35. Rockafellar, R.T., Wets, J.-B.: Variational analysis. Grundlehren der Mathematischen Wissenschaften [Fundamental Principles of Mathematical Sciences], vol. 317. Springer-Verlag, Berlin (1998)

36. Shvartsman, P.: On Lipschitz selections of affine-set valued mappings. Geom. Funct. Anal. 11(4), 840-868 (2001)

37. Shvartsman, P.: Lipschitz selections of set-valued mappings and Helly's theorem. J. Geom. Anal. 12(2), 289-324 (2002)

38. Shvartsman, P.: Barycentric selectors and a Steiner-type point of a convex body in a Banach space. J. Funct. Anal. 210(1), 1-42 (2004)

39. Smajdor, A.: Additive selections of superadditive set-valued functions. Aequationes Math. 39, 121-128 (1990)

40. Smajdor, A., Smajdor, W.: Affine selections of convex set-valued functions. Aequationes Math. 51, 12-20 (1996)

41. Wąsowicz, Sz.: On affine selections of set-valued functions. J. Appl. Anal. 1(2), 173-179 (1995)

42. Zaslavski, A.J.: On the existence of a linear selection of a superlinear multivalued mapping. Mathematicheskie Zametki 29, 557-566 (1981) (in Russian) 